Bull. Iraq nat. Hist. Mus.

https://doi.org/10.26842/binhm.7.2019.15.3.0319

June, (2019) 15 (3): 319-333

\title{
A SURVEY OF WEEVILS (COLEOPTERA, CURCULIONOIDEA) FROM SOME LOCALITES OF KURDISTAN REGION- IRAQ, WITH NEW RECORDS TO THE ENTOMOFAUNA OF IRAQ
}

\author{
Halgurd Rashed Ismael Akrawi* and Talal Tahir Mahmoud \\ Duhok University, College of Agriculture, Department of Plant Protection, \\ Kurdistan Region, Iraq \\ *Corresponding author e-mail: halgurd.ismael@uod.ac
}

Received Date: 12 January 2019, Accepted Date: 27 March 2019, Published Date: 27 June 2019

\begin{abstract}
This work is the first study of the Curculionoidea fauna from Kurdistan region of Iraq, based on the intensive survey in different localities of Kurdistan from March 2016 to November 2017. In total, 41 species belonging to 28 genera, 21 tribes and 3 families were collected and identified, including 25 species newly recorded for the Iraqi fauna.
\end{abstract}

General distribution, collecting localities and methods, with plant association data for each species are given.

Keywords: Coleoptera, Curculionoidea, Entomofauna, Iraq, Kurdistan.

\section{INTRODUCTION}

The superfamily Curculionoidea, commonly named snout beetles or weevils, is one of the highest diversity group of insects and probably the largest family of the order Coleoptera, that including over 62,000 species and around 6,000 genera thus far described (Oberprieler et al., 2007). They are small to large-sized beetles (approximately 1-60 mm) of different shapes, colors and habitats; Weevils can be recognized by their more or less long and slender rostrum with mouthparts situated at its apex, and mostly geniculate antennae with more or less compact antennal club (Thompson, 1992; Kuschel, 1995; Alonso- Zarazaga and Lyal, 1999; Anderson, 2002; Marvaldi et al., 2002; Oberprieler et al., 2007). These beetles are phytophagous insects exhibiting narrow to broad oligophagy or polyphagy; Most species develop on living plants whereas some others are feeding on decaying materials, some species are important as a medical insect causes allergic skin, rhinitis and asthma to bakers and laboratory workers such as Sitophilus species. Adults and larvae feed on plant roots, stems, leaves and seeds. Generally, females bore into various parts of plants where eggs are laid. Some weevils are important pests of agricultural crops, ornamental plants and stored products as well as trees and forestry products. On the other hand, a few species of weevils were successfully used in the biological control as an effective method for weed control (Wibmer and O'Brein, 1986; Herling et al., 1995; Anderson, 2002; Oberprieler, 2004).

The Iraqi fauna of curculionids is poorly studied, especially that of the Kurdistan region, in addition to taxa listed in the newest catalogue (Alonso-Zarazaga et al., 2017); the records of Curculionoidea in Iraq were quoted in the official lists published by Derwesh $(1963,1965)$ 


\section{A survey of weevils (Coleoptera, Curculionoidea)}

who listed 25 and 40 species respectively, Shalaby et al. (1966) who recorded one species, Kaddou (1967) who reported 11 species, Khalaf and Al-Omor (1974) who listed 23 species, Abdul-Rassoul (1976) who recorded 25 species, and Al-Ali (1977) who listed 28 species. Thereafter, Salih (2007) studied 20 species of two subfamilies, Abul Wahed and Alhadalg (2015) recorded 2 additional species from Basrah province, and Ismail (2015) studied microsculpture of 8 species and 3 genera. With the species recorded in this note, the Iraqi fauna of Curculionoidea has reached 85 species, surely far less of those actually occurring in this large and diverse country.

This paper represents the first study of the weevils in Kurdistan Region, Iraq; and the aim of this study is faunistic study of weevils to contribute the knowledge of this superfamily in Iraq.

\section{MATERIALS AND METHODS}

In this study, the specimens were collected in various localities of Iraqi Kurdistan: Erbil, Sulaymaniyah and Duhok provinces, from March 2016 to November 2017; the specimens were collected using different methods, such as sweeping net, beating sheet, pooter aspirator, light trap and hand-picking, from fruit and forest trees, vegetables and wild plants during 3-4 field collecting trips per week in the spring (March, April and May) and summer months (June, July and August); while we made two trips for the other months.

All specimens collected by the first author are part of a $\mathrm{Ph}$. D. study focused on the faunistic study of the Curculionoidea of Iraqi Kurdistan; all specimens are preserved in the Museum of Agriculture College, Duhok University, each of them bearing information about the place and date of collecting. Most of the species mentioned in this paper have been identified by Enzo Colonnelli, of Museo di Zoological, Sapienza Universita di Roma, Italy.

Taxonomic position and scientific names of others species were identified by authors using original descriptions, available keys and catalogues, such as those by Zherikhin and Egorov (1991), May (1993), Alonso-Zarazaga and Lyal (1999, 2002), Colonnelli (2003, 2004), Legalov (2006, 2011), Lyal and Alonso-Zarazaga (2006), Velázquez de Castro et al. (2007) and Löbl and Smetana (2011, 2013). Tribes, genera, and species of the records have been listed alphabetically, higher taxonomy of the newest classification of Curculioniodea and general distribution of each species primarily follow Alonso-Zarazaga et al. (2017).

The identification of species that have not been recorded for Iraq in Alonso-Zarazaga et al. (2017) was confirmed by Mohammad Saleh Abdul-Rassoul, God bless his soul, from Iraq Natural History Research center and Museum, University of Baghdad. The collected plants were identified by Saleem Ismail Shahbaz of the University of Duhok, College of Agriculture.

Abbreviations of depositories: The names of collections and museums that provided species of this study are abbreviated as follows: MCZR = Museo Civico di Zoologia, Rome, Italy; MAMR = Ministry of Agriculture, Museum of Directorate Agricultural Research; UODM = University of Duhok, Museum of Agriculture College.

\section{RESULTS}

The result of this investigation is a total of 41 species belonging to 3 families, 9 subfamilies, 21 tribes, and 28 genera of Curculionoidea collected and identified from Kurdistan region. The list of the collected species is given alphabetically below: 
Halgurd Rashed Ismael Akrawi and Talal Tahir Mahmoud

1- Family: Attelabidae Billberg, 1820

Subfamily: Attelabinae Billberg, 1820

Tribe: Attelabini Billberg, 1820

Attelabus sulcifrons (Argod-Vallon, 1895)

Material examined: Duhok (Akre, Atrish) and Erbil (Mergasor), May 2016 and 2017, on

Quercus aegilops.

Collecting methods: Beating and hand-picking.

General distribution: Armenia, Bulgaria, Georgia, Greece, Macedonia, Turkey and Syria; newly record to Iraq.

Subfamily: Rhynchitinae Gistel, 1848

Tribe : Rhynchitini Gistel, 1848

Rhynchites smyrnensis Desbrochers des Loges, 1869

Materials examined: Duhok :Amediand Kanimasy, April 2016 and 2017, on apricot and pear trees.

Collecting methods: Beating, hand-picking and light trap jointed to trees.

General distribution: Greece, Iran, Jordan, Syria, Turkmenistan and Turkey; newly record to Iraq.

Rhynchites trojanus Gyllenhal, 1839

Materials examined: Duhok: Zawita April and May 2017, on apricot tree.

Collecting methods: Beating, hand-picking and light trap.

General distribution: Cyprus, Greece, Jordan and Turkey; newly record to Iraq.

2- Family: Brentidae Billberg, 1820

Subfamily: Apioninae Schoenherr, 1823

Tribe: Apionini Schoenherr, 1823

Apion frumentarium (Linnaeus, 1758)

Materials examined: Duhok: Sumel, June and July 2016, on Rumex crispus.

Collecting methods: Sweeping net.

General distribution: Western Palaearctic, recorded from Afghanistan, Algeria, Armenia, Austria, Azerbaijan, Bosnia, Bulgaria, Cyprus, Croatia, Czech Republic, Denmark, Estonia, France, Germany, Georgia, Great Britain, Greece, Hungary, Iran, Ireland, Italy, Jordan, Kazakhstan, Latvia, Lichtenstein, Lithuania, Luxembourg, The Netherlands, Norway, Poland, Portugal, Romania, European Russia, Serbia, Slovakia, Spain, Sweden, Switzerland, Syria, Turkey, Ukraine; newly record to Iraq.

Tribe: Aspidapiini Alonso-Zarazaga, 1990

Aspidapion radiolus (Marsham, 1802)

Materials examined: Duhok :Atrish ; Sulaymaniyah :Dukan, July 2016 and 2017, on

Malva neglecta, Alcea setosa and A. kurdica.

Collecting methods: beating and aspirator.

General distribution: Widely distributed in Western Palaearctic, reported from Albania, Algeria, Afghanistan, Armenia, Austria, Azores, Azerbaijan, Bulgaria, Canary Islands, Croatia, Cyprus, Czech Republic, Germany, Denmark, Estonia, Finland, Georgia, Great Britain, Greece, Hungary, Iraq, Italy, Jordan, Kazakhstan, Latvia, Lebanon, Libya, Lichtenstein, Luxembourg, Madeira, Malta, Morocco, The Netherlands, Norway, Portugal, 


\section{A survey of weevils (Coleoptera, Curculionoidea)}

Poland, Romania, Russia, Serbia, Spain, Slovakia, Switzerland, Sweden, Syria, Tadzhikistan, Turkey, Tunisia, Ukraine and Uzbekistan; this species reported also from tropical Africa.

Aspidapion aeneum (Fabricius, 1775)

Materials examined: Duhok: Akre and Atrish; Erbil :Shaqlawa, July and August 2017, on

Malva neglecta and Alcea setosa.

Collecting methods: Beating and aspirator.

General distribution: Western Palaearctic, recorded from Albania, Algeria, Afghanistan, Armenia, Azerbaijan, Bulgaria, Cyprus, Croatia, Czech Republic, Germany, Denmark, France, Georgia, Great Britain, Hungary, Greece, Iran, Italy, Kazakhstan, Kyrgyzstan, Lebanon, Lithuania, Macedonia, Malta, Morocco, The Netherlands, Portugal, European, Russia, Serbia, Spain, Slovakia, Switzerland, Sweden, Syria, Tunisia, Ukraine and Uzbekistan; new record to Iraq.

Tribe : Malvapiini Alonso-Zarazaga, 1990

Rhopalapion longirostre (Olivier, 1807)

Materials examined: Duhok: Zawita and Kanimasy August 2016, on Malva neglecta and M. anicaeensis.

Collecting methods: Beating and aspirator.

General distribution: Widespread in Western Palaerctic, introduced to North America, occurring in Albania, Algeria, Afghanistan, Austria, Armenia, Austria, Azerbaijan, Bulgaria, Croatia, Cyprus, Czech Republic, Germany, France, Georgia, Greece, Hungary, Iraq, Iran, Italy, Jordan, Kazakhstan, Lebanon, Luxembourg, Montenegro, The Netherlands, Poland, Romania, South European, Russia, Serbia, Spain, Slovakia, Syria, Switzerland, Syria, Tadzhikistan, Turkmenistan, Turkey, Uzbekistan and Ukraine; newly record to Iraq.

Tribe: Oxystomatini Alonso-Zarazaga, 1990

Cyanapion pseudarrogans (Reitter, 1901)

Material examined: Duhok: Amedi and Bamarny May and June 2016, on Medicago polymorpha and $M$. rigidula.

Collecting methods: Sweeping net.

General distribution: Kazakhstan and Uzbekistan; newly record to Iraq.

Eutrichapion arrogans (Wencker, 1858)

Materials examined: Duhok: Bamarny and Sarsenk June and July 2017, on Medicago radiate and $M$. rigidula.

Collecting methods: Beating and sweeping net.

General distribution: Jordan, Syria and Turkey; newly record to Iraq.

Tribe : Piezotrachelini Voss, 1959

Protapion assimile (Kirby, 1808)

Materials examined: Duhok: Zakho and Zawita; Erbil: Soran and Mergasor, June 2017, on Trifolium pilulare and T. stellatum.

Collecting methods: Beating, sweeping and light trap.

General distribution: Western Palaearctic, recorded from Algeria, Albania, Armenia, Azerbaijan, Belgium, Bulgaria, Croatia, Czech Republic, Denmark, Germany, France, Finland, Georgia, Great Britain, Greece, Hungary, Iran, Italy, Ireland, Latvia, Lichtenstein, Lithuania, Luxembourg, The Netherlands, Norway, Portugal, Poland, Serbia, Slovakia, Spain, Sweden, Switzerland, Syria, Russia, Turkey, Tunisia and Ukraine; newly record to Iraq. 
3- Family : Curculionidae Latreille, 1802

Tribe: Baridini Schoenherr, 1836

Malvaevora timida (Rossi, 1792)

Material examined: Duhok :Deralok and Kanimasy, August 2017, on Malva neglecta.

Collecting methods: Beating and aspirator.

General distribution: Western Palaearctic, reported from Algeria, Austria, Bulgaria, Cyprus, France, Iran, Italy, Hungary, Libya, Kazakhstan, Malta, Morocco, Slovakia, Slovenia, Spain, South European, Romania, Russia, Switzerland, Tunisia, Turkey and Turkmenistan; newly record to Iraq.

Subfamily: Curculioninae Latreille, 1802

Tribe: Cionini Schoenherr, 1825

Cionus olivieri Rosenschoeld, 1838

Materials examined: Duhok: Kanimasy, August and September 2016, on Verbascum thapsus and V. speciosum.

Collecting methods: Beating and sweeping.

General distribution: Widespread in Western Palaearctic and Central Asia, recorded from: Afghanistan, Andorra, Albania, Austria, Armenia, Bosnia, Belgium, Cyprus, Croatia, Czech Republic, Germany, France, Estonia, Herzegovina, Hungary, Georgia, Greece, Iran, Latvia, Italy, Kirgizstan, Poland, Macedonia, Montenegro, Serbia, South European, Slovenia Russia, Slovakia, Syria, Romania, Tadzhikistan, Turkey, Turkmenistan and Uzbekistan. Iraq (Derwesh, 1963).

Tribe: Curculionini Latreille, 1802

Curculio elephas (Gyllenhal, 1835)

Materials examined: Sulaymaniyah: Peramagrom, May 2017, on Quercus infectoria.

Collecting methods: Beating and light trap.

General distribution: Europe and Mediterranean reported from Albania, Algeria, Austria, Belgium, Bosnia, Bulgaria, Germany, Croatia, Czech Republic, Cyprus, Greece, France, Herzegovina, Hungary, Italy, Luxembourg, Morocco, Macedonia, Poland, Romania, Russia, South European, Spain, Slovakia, Switzerland, The Netherlands, Turkey and Tunisia; newly record to Iraq.

Tribe: Mecinini Gistel, 1848

Rhinusa asellus (Gravenhorst, 1807)

Materials examined: Duhok: Qasrok and Bablo, May 2016 and 2017, on Verbascum speciosum.

Collecting methods: Beating and sweeping.

General distribution: Austria, Belgium, Bulgaria, Czech Republic, Germany, France, Greece, Italy, Luxembourg, Hungary, Macedonia, Moldavia, Poland, The Netherlands, Romania, Russia, South European, Slovakia, Spain, Switzerland, The Netherlands and Turkey; newly record to Iraq.

Rhinusa bipustulata (Rossi, 1792)

Materials examined: Duhok :Bablo and Chamanky, May and June 2017, on Verbascum thapsus and V. speciosum.

Collecting methods: Beating and sweeping.

General distribution: Western Palaearctic, recorded from Afghanistan, Albania, Austria, Armenia, Azerbaijan, Bosnia, Belgium, Bulgaria, Byelorussia, Croatia, Czech Republic, France, Greece, Georgia, Herzegovina, Hungary, Lebanon, Italy, Moldavia, Montenegro, 


\section{A survey of weevils (Coleoptera, Curculionoidea)}

Romania, Russia, Portugal, Poland, South European, Slovakia, Slovenia, Serbia, Syria, Switzerland, Turkey, Turkmenistan, Uzbekistan and Ukraine; newly record to Iraq.

Tribe: Tychiini C. G. Thomson, 1859

Tychius meliloti Stephens, 1831

Materials examined: Duhok: Sarsenk and Bamarny; Erbil: Choman, June 2016 and 2017, on

Melilotus alba and M. indicus.

Collecting methods: Sweeping net.

General distribution: Widely distributed across Palaearctic and introduced to North America and tropical Africa, recorded from Afghanistan, Albania, Algeria, Austria, Armenia, Azerbaijan, Belgium, Bosnia, Bulgaria, Byelorussia, China, Croatia, Czech Republic, Denmark, Germany, Estonia, Finland, France, Georgia, Great Britain, Greece, Herzegovina, Hungary, Italy, Iran, Kyrgyzstan, Kazakhstan, Latvia, Lithuania, Lichtenstein, Malta, Macedonia, Moldavia, Mongolia, Montenegro, Norway, Portugal, Poland, Romania, Russia, Serbia, Slovenia, Slovakia, Spain, Sweden, Switzerland, Tadzhikistan, Turkey, Turkmenistan, Ukraine and Uzbekistan; newly record to Iraq.

Tychius pusillus Germar, 1842

Materials examined: Duhok: Mangeshky and Bamarny; Erbil: Choman, June and July 2017, on Trifolium purpureum and T. stellatum.

Collecting methods: Sweeping net.

General distribution: Europe and Mediterranean, introduced to tropical Africa, reported from Algeria, Albania, Austria, Belgium, Bulgaria, Croatia, Czech Republic, Germany, France, Great Britain, Greece, Hungary, Italy, Lichtenstein, Malta, Montenegro, Morocco, Portugal, Poland, South European, Romania, Russia, Slovakia, Spain and Switzerland; newly recorded to Iraq.

Tychius stephensi Schoenherr, 1835

Materials examined: Duhok (Sarsenk, Qadeshy) June and July 2017, on Trifolium resupinatum.

Collecting methods: Sweeping net.

General distribution: Palaearctic, recorded from Albania, Austria, Armenia, Azerbaijan, Belgium, Bosnia, Bulgaria, Byelorussia, Canary Islands, Croatia, Czech Republic, Germany, Denmark, Estonia, Finland, France, Georgia, Greece, Great Britain, Herzegovina, Hungary, Ireland, Iran, Italy, Kazakhstan, Jordan, Latvia, Lebanon, Lichtenstein, Lithuania, Luxembourg, Malta, Macedonia, Moldavia, Montenegro, Morocco, Norway, Portugal, Poland, Serbia, Spain, Slovakia, Slovenia, Sweden, Syria, Switzerland, Romania, Russia and Ukraine; newly recorded to Iraq.

Subfamily: Ceutorhynchinae Gistel, 1848

Tribe: Ceutorhynchini Gistel, 1848

Ceutorhynchus fallax Boheman, 1845

Materials examined: Duhok: Khanky, March 2017, on Sinapis alba and Brassica nigra. Collecting methods: Beating and aspirator.

General distribution: Algeria, Azerbaijan, Armenia, Bulgaria, France, Georgia, Greece, Italy, Jordan, Moldavia, Morocco, Romania, Russia, South European, Spain, Slovakia, Syria, Turkey and Tunisia; newly record to Iraq.

Hadroplontus trimaculatus (Fabricius, 1775)

Materials examined: Duhok (Dargaly) and Erbil (Rawanduz), April 2016 and 2017, on Carduus pycnocephalus and Notobasis syriaca. 
Collecting methods: Sweeping net.

General distribution: Western Palaearctic; reported from Albania, Algeria, Austria, Armenia, Azerbaijan, Belgium, Bosnia, Bulgaria, Croatia, Czech Republic, Cyprus, Germany, France, Georgia, Great Britain, Greece, Herzegovina, Hungary, Iraq, Italy, Iran, Luxembourg, Moldavia, Morocco, The Netherlands, Portugal, Poland, Romania, Russia, South European, Slovenia, Slovakia, Switzerland, Spain, Syria, Tunisia and Ukraine; newly record to Iraq.

Subfamily: Entiminae Schoenherr, 1823

Tribe : Brachyderini Schoenherr, 1826

Pholicodes fausti Reitter, 1890

Materials examined: Duhok: Akre and Bjil ; Sulaymaniyah: Qaladezy, May 2016 and 2017, on Prosopis farcta.

Collecting methods: Beating and hand-picking

General distribution: Iran, Iraq, Turkey

Tribe: Cyphicerini Lacordaire, 1863

Myllocerus damascenus Miller, 1861

Materials examined: Duhok: Dinarta and Grbish; Erbil: Sherwanmazn, July and August 2016, on Ceratonia siliqua.

Collecting methods: Beating.

General distribution: Armenia, Cyprus, Lebanon, Syria, Turkey and tropical Africa. Recorded in Iraq (Derwesh, 1963)

Tribe: Phyllobiini Schoenherr, 1826

Phyllobius nudiamplus Reitter, 1916.

Materials examined: Duhok: Atrish, Sarsenk and Blye; Erbil: Hiran and Mergasor; Sulaymaniyah: Dukan, Rania and Qaladezy, April and May 2017, on Quercus aegilops.

Collecting methods: Beating and aspirator.

General distribution: Turkey; newly record to Iraq.

Note. This is the first finding of this species after its description (Reitter, 1916) with precise collecting data.

Tribe: Polydrusini Schoenherr, 1823

Polydrusus virbius Reitter, 1899

Materials examined: Duhok: Sumel and Zawita; Erbil: Shaqlawa and Hiran; Sulaymaniyah: Rania April and May 2017, on apple, apricot, peach trees and on Quercus aegilops and $Q$. infectoria.

Collecting methods: beating, aspirator and light trap.

General distribution: Iran and Turkmenistan; newly record to Iraq.

Tribe: Sitonini Gistel, 184

Charagmus intermedius (Küster, 1847)

Materials examined: Duhok: Dargaly, June 2016, on Hippocrepis unisiliquosa.

Collecting methods: Sweeping net.

General distribution: Algeria, Belgium, Bosnia, Bulgaria, Croatia, Cyprus, Germany, France, Greece, Iran, Italy, Herzegovina, Lebanon, Luxembourg, Madeira, Malta, Macedonia, Morocco, Montenegro, Portugal, Spain, Syria Switzerland, Tunisia and Turkey; newly record to Iraq. 


\section{A survey of weevils (Coleoptera, Curculionoidea)}

Sitona lividipes Fåhraeus, 1840

Materials examined: Duhok: Batifa and Kamaka; Sulaymaniyah: Bshdar, May and June 2017, on Medicago polymorpha and M. orbicularis.

Collecting methods: Sweeping net.

General distribution: Algeria, Bulgaria, Egyptian, France, Italy, Iran, Greece, Lebanon, Portugal, Macedonia, Morocco, Spain, Syria, Turkey and Tunisia; newly record to Iraq.

Sitona macularius (Marsham, 1802)

Material examined: Duhok: Bany and Siarateka; Erbil: Choman and Hajiumaran, May and June 2017, on Medicago polymorpha and M. rigidula.

Collecting methods: Sweeping net.

General distribution: Palaearctic, recorded from Afghanistan, Algeria, Albania, Armenia, Austria, Azerbaijan, Belgium, Bosnia, Bulgaria, Byelorussia, Canary Islands, Croatia, Cyprus, Czech Republic, Germany, Denmark, Egyptian, Estonia, Finland, France, Georgia, , Great Britain, Greece, Herzegovina, Hungary, Iran, Iraq, Italy, Jordan, Kazakhstan, Kirgizstan, Latvia, Lebanon, Lithuania, Libya, Luxembourg, Malta, Macedonia, Morocco, Moldavia, Pakistan, Portugal, Poland, Romania, Russia, Saudi-Arabia, Serbia, Slovakia, Slovenia, Spain, Syria, Switzerland, The Netherlands, Tadzhikistan, Turkey, Tunisia, Turkmenistan and Ukraine.

Sitona puncticollis Stephens, 1831

Materials examined: Duhok: Siarateka, Chamanky and Bablo; Erbil: Harir and Soran, April and May 2016, on Medicago rigidula and Trifolium pilulare.

Collecting methods: Sweeping net.

General distribution: Algeria, Albania, Austria, Azerbaijan, Azores, Belgium, Bosnia, Bulgaria, Byelorussia, Croatia, Cyprus, Czech Republic, Germany, Denmark, Egyptian, Estonia, Faeroe Islands, Finland, France, Georgia, Greece, Great Britain, Herzegovina, Hungary, Italy, Iran, Ireland, Kazakhstan, Latvia, Lithuania, Libya, Luxembourg, Macedonia, Morocco, Norway, Portugal, Poland, Romania, Russia, Serbia, Slovakia, Slovenia, Sweden, Spain, Syria, Switzerland, The Netherlands, Tadzhikistan, Turkey, Tunisia, Ukraine and Uzbekistan; newly record to Iraq.

Subfamily: Lixinae Schoenherr, 1823

Tribe: Cleonini Schoenherr, 1826

Coniocleonus excoriatus (Gyllenhal, 1834)

Materials examined: Duhok: Akre and Hashka, September 2017, on grass and crawling on the ground.

Collecting methods: Hand-picking.

General distribution: Austria, Belgium, Bulgaria, Canary Islands, Cyprus, Czech Republic, Egyptian , France, Germany, Greece, Hungary, Iran, Iraq, Italy, Jordan, Libya, Malta, Macedonia, Morocco, Pakistan, Portugal, Russia, Slovakia, Spain, Sweden, Switzerland, Syria, Turkey, Tunisia, Ukraine and Yemen; Iraq (Derwesh, 1965).

Coniocleonus nigrosuturatus (Goeze, 1777)

Material examined: Duhok: Grbishm, Dinarta and Bakrman, June 2016 and 2017, on Erodium cicutarium.

Collecting methods: Hand-picking and sweeping.

General distribution: Western Palaearctic and Oriental Region, reported from Afghanistan, Algeria, Albania, Astoria, Armenia, Azerbaijan, Belgium, Bulgaria, Croatia, Cyprus, Czech Republic, Germany, Egyptian, France, Georgia, Greece, Hungary, Italy, Iran, Iraq, Libya, Jordan, Kazakhstan, Kirgizstan, Lebanon, Moldavia, Morocco, Pakistan, Portugal, Romania, 
Halgurd Rashed Ismael Akrawi and Talal Tahir Mahmoud

Russia, Serbia, Slovenia, Slovakia, Spain, Syria, Switzerland, Tadzhikistan, Turkey, Turkmenistan, Ukraine and Uzbekistan.

Coniocleonus pseudobliquus (J. Müller, 1921)

Materials examined. Duhok: Mangeshky, Zawita and Khansye, August 2016, on grass and under stones.

Collecting methods: Hand-picking.

General distribution: Bosnia, Bulgaria, Croatia, Hercegovina, Greece, Iraq, Malta, Macedonia, Romania, Serbia, Slovenia, Turkey and Ukraine.

Temnorhinus brevirostris (Gyllenhal, 1834)

Materials examined: Duhok: Amedi and Chamanky; Erbil: Soran, May and June 2017, on Salsola vermiculata and $S$. crassa.

Collecting methods: Beating and sweeping net.

General distribution: Algeria, Canary Islands, Cyprus, Egyptian, France, Iraq, Iran Italy, Jordan, Libya, Kazakhstan, Malta, Morocco, Oman, Pakistan, Saudi Arabia, Spain, Turkey, Tunisia and Yemen; known also from Afro-tropical and Oriental Region.

Tribe: Lixini Schoenherr, 1823

Bangasternus orientalis (Capiomont, 1873)

Materials examined: Duhok: Bardarash and Akre; Erbil: Khabat and Koysinjaq, March and April 2016, on Centaurea hyalolepis and C. solstitialis.

Collecting methods: Beating and aspirator.

General distribution: Western Palaearctic and introduced in North America, reported from Afghanistan, Armenia, Austria, Azerbaijan, Bulgaria, Cyprus, Egyptian, Georgia, Greece, Hungary, Iraq, Iran, Italy, Jordan, Kazakhstan, Lebanon, Macedonia, Romania, European Russia, Syria, Slovakia, Turkey, Tajikistan and Uzbekistan.

Larinus latus (Herbst, 1783)

Materials examined: Duhok: Bakrman, Atrish, Zakho and Amedi; Erbil: Shaqlawa, Rawanduz and Mergasor; Sulaymaniyah: Dukan, Sharbazher and Halabja, April and May 2016 and 2017, on Onopordum carduchorum.

Collecting methods: Beating and hand-picking.

General distribution: Palaearctic, introduced to the Australian Region, recorded from Albania, Austria, Armenia, Azerbaijan, Bosnia, Bulgaria, Croatia, Cyprus, Germany, France, Georgia, Greece, Herzegovina, Hungary, Italy, Iran, Moldavia, Montenegro, Russia, Romania, Serbia, Slovakia, Slovenia, Syria, Turkey and Ukraine. This species was reported to Iraq by Derwesh (1965).

Larinus onopordi (Fabricius, 1787)

Materials examined: Duhok: Kanimasym, Sarsenk and Khanky; Erbil :Harir and Khalifan, Hiran; Sulaymaniyah: Piramagrom, June and July 2016 and 2017, on Echinops ritro and Onopordum carduchorum.

Collecting methods: beating and hand-picking.

General distribution: Western Palaearctic and tropical Africa, recorded from Algeria, Armenia, Azerbaijan, Bosnia, Bulgaria, Cyprus, Egyptian, France, Georgia, Greece, Herzegovina, Italy, Iran, Kazakhstan, Libya, Lebanon, Morocco, Portugal, Russia, Saudi Arabia, Serbia, Spain, Syria, Tadzhikistan, Turkey, Tunisia, Turkmenistan and Ukraine. Reported to Iraq by Derwesh (1963). 
A survey of weevils (Coleoptera, Curculionoidea)

Larinus grisescens Gyllenhal, 1835

Materials examined: Duhok: Dinarta and Bjil; Erbil: Mergasor and Piran, April 2016 and 2017, on Silybum marianum.

Collecting methods: Beating and hand-picking.

General distribution: Albania, Bulgaria, Cyprus, Gerrce, Georgia, Italy, Iraq, Iran, Lebanon, Macedonia, Moldavia, Montenegro, Russia, Serbia, Syria, Turkey.

Larinus ovaliformis Capiomont, 1874

Materials examined: Duhok: Akre, Amedi, Atirsh, Sumel and Bardarash; Erbil: Khabat, Kory, Shaqlawa, Harir and Koysinjaq; Sulaymaniyah: Dukan, Rania, Pshdar, Piramagrom and Halabja, March and April 2016 and 2017, on Silybum marianum and Notobasis syriaca.

Collecting methods: Beating and hand-picking.

General distribution: Cyprus, Iran, Lebanon, Libya, South European Russia, Syria and Turkey. Iraq (Derwesh, 1963).

Lixus cardui Olivier, 1807

Materials examined: Duhok: Bakrman, Atrish, Zakho and Amedi; Erbil: Shaqlawa, Rawanduz and Mergasor; Sulaymaniyah: Dukan and Halabja, April and May 2016 and 2017 on Onopordum carduchorum and Carduus pycnocephalus.

Collecting methods: Beating and hand-picking.

General distribution: Algeria, Austria, Armenia, Azerbaijan, Bosnia, Bulgaria, Croatia, Cyprus, Czech Republic, France, Georgia, Germany, Greece, Hercegovina, Hungary, Iran, Italy, Moldavia, Morocco, Poland, Romania, Portugal, Russia,Syria, Slovakia, Spain, Turkey and Ukraine. Iraq (Khalaf and Al-Omor, 1974).

Subfamily: Hyperinae Marseul, 1863

Tribe: Hyperini Marseul, 1863

Hypera nigrirostris (Fabricius, 1775)

Material examined: Duhok: Zawita, Mangeshky and Bamarny, March 2016 and 2017, on

Medicago rigidula and Trifolium campestre.

Collecting methods: Sweeping and light trap.

General distribution: Palaearctic, introduced to North America, reported for Albania, Armenia, Azerbaijan, Austria, Belgium, Bosnia, Bulgaria, Byelorussia, China, Croatia, Cyprus, Czech Republic, Denmark, Estonia, Finland, France, Georgia, Germany, Great Britain, Greece, Herzegovina, Hungary, Ireland, Italy, Japan, Kazakhstan, Latvia, Lithuania, Luxembourg, Malta, Macedonia, Moldavia, Montenegro, Morocco, Norway, Portugal, Poland, Romania, Russia, Serbia, Slovakia, Slovenia, Spain, Sweden, Switzerland, Syria, The Netherlands, Turkey and Ukraine; newly record to Iraq.

Hypera postica (Gyllenhal, 1813)

Materials examined: Duhok: Akre and Bakrman; Erbil :Shaqlawa an Hiran; Sulaymaniyah: Dukan, Qaladze and Piramagrom, April and May 2016 and 2017, on Trifolium campestre and T. resupinatum.

Collecting methods: Sweeping net and light trap.

General distribution: Palaearctic, introduced to North America, reported from Afghanistan, Algeria, Albania, Armenia, Austria, Azerbaijan, Belgium, Bosnia, Bulgaria, Byelorussia, Canary Islands, Northern China, Croatia, Cyprus, Czech Republic, Denmark, Germany, Egyptian, Estonia, Finland, France, Georgia, Greece, Great Britain, Herzegovina, Hungary, Iraq, Iran, Italy, Japan, Kazakhstan, Kirgizstan, Libya, Latvia, Lithuania, Luxembourg, Malta, Macedonia, Madeira, Moldavia, Mongolia, Montenegro, Morocco, Norway, Poland, Portugal, 
Halgurd Rashed Ismael Akrawi and Talal Tahir Mahmoud

Romania, Russia, Slovakia, Serbia, Slovenia, Spain, South Korea, Sweden, Syria, Switzerland, The Netherlands, Turkey, Tunisia, Turkmenistan, Ukraine and Uzbekistan.

Hypera venusta (Fabricius, 1791)

Materials examined: Duhok: Amedi and Deralok, May 2017, on Trifolium campestre.

Collecting methods: Sweeping net and light trap.

General distribution: Algeria, Armenia, Azerbaijan, Austria, Belgium, Bosnia, Bulgaria, Byelorussia, Croatia, Cyprus, Czech Republic, Denmark, Estonia, France, Germany, Great Britain, Greece, Herzegovina, Hungary, Italy, Ireland, Libya, Kazakhstan, Latvia, Lithuania, Luxembourg, Macedonia, Malta, Montenegro, Morocco, Norway, Poland, Portugal, Romania, Russia, Slovenia, Serbia, Slovakia, Spain, Sweden, Syria, Switzerland, Turkey, Tunisia and Ukraine; newly record to Iraq.

\section{DISCUSSION}

This study is the result of the field survey carried out in the Kurdistan region of Iraq focusing on weevils, here we list 41 species of which 25 were hitherto not recorded from Iraq, and 16 species previously quoted in the lists of the Palaearctic region and lists of Iraqi fauna. Hence, the total of $110 \mathrm{spp}$. of the three families is currently known from Iraq. The primeval and semi-primeval vegetation of Iraqi Kurdistan is very rich as plant biodiversity concerns. Kurdistan deserves further, more comprehensive entomological investigation, which will certainly contribute by many new records to the better knowledge of Iraqi weevil fauna including findings of new species to the science. The climate of Kurdistan is very favorable, and serves as a refuge for plants and animals. Further studies may also lead to the discovery of host plants and biology of several poorly known species improving our knowledge about the aspects of their life cycle, environmental conditions and needs of the nature conservation.

\section{ACKNOWLEDGEMENTS}

We are extremely grateful to Dr. E. Colonnelli (A.R.D.E. Museo Civico di Zoologia, Rome, Italy) for the identification of most collected specimens and to Dr. G. Sabatinelli (Natural History Museum of Geneva, Switzerland) for important scientific cooperation and great role in progress of our research. Many thanks also to Dr. M.S. Abdul-Rassoul (God bless his soul) and Dr. Razzaq Shalan Augul, Iraq Natural History Research Center and Museum, University of Bagdad, for their confirming of unrecorded species in Iraq.

\section{LITERATURE CITED}

Abdel Wahed, M. A. and Alhadalg, K. S. H. 2015. Two billbugs (Curculionidae; Coleoptera), first recorded from Iraq. Journal of Purity, Utility Reaction and Environment, 4(6): 190-193.

Abdul-Rassoul, M. S. 1976. Checklist of Iraq Natural History Museum insects collection. Natural History Research Center, Publication, 30: 1- 41.

Al-Ali, A. S. 1977. Phytophagous and entomophagous insects and mites in Iraq. Natural History Research Center, Publication, 33: 1-142.

Alonso-Zarazaga, M. A., Barrios, H., Borovec, R., Bouchard, P., Caldara, R., Colonnelli, E., Gültekin, L., Hlavač, P., Korotyaev, B., Lyal, C.H.C., Machado, A., Meregalli, M., Pierotti, H., Ren, L., Sanchez-Ruiz, M., Sforzi, A., Silfverberg, H., Skuhrovec, J., Tryzna, M., Velázquez de Castro, A. J. and Yunakov, N. N. 2017. Cooperative 
A survey of weevils (Coleoptera, Curculionoidea)

catalogue of Palaearctic Coleoptera Curculionoidea. Monografías electrónicas de la Sociedad Entomológica Aragonesa, 8: 1-729.

Alonso-Zarazaga, M. A. and Lyal, C. H. C. 1999. A world catalogue of families and genera Curculionoidea (Insecta: Coleoptera) (excepting Scolytidae and Platypodidae). Entomopraxis, Barcelona, 315 pp.

Alonso-Zarazaga, M. A. and Lyal, C. H. C. 2002. Addenda and corrigenda to 'A world catalogue of families and genera of Curculionoidea (Insecta: Coleoptera). Zootaxa, 63 : $1-37$.

Anderson, R. S. 1995. An evolutionary perspective of diversity in Curculionoidea. Memoirs of the Entomological Society of Washington, 14: 103-114.

Anderson, R. S. 2002. Curculionidae Latreille 1802 (pp. 722-815). In: Arnett Jr., R. H., Thomas, M. C., Skelley, P. E. and Frank, J. H. (eds). American Beetles. Vol. 2, Polyphaga: Scarabaeoidea through Curculionoidea CRC Press, Boca Raton, xiv, 816 pp.

Colonnelli, E. 2003. A revised checklist of Italian Curculionoidea (Coleoptera). Zootaxa, 337: $1-142$.

Colonnelli, E. 2004. Catalogue of Ceutorhynchinae of the world, with a key to genera (Insecta: Coleoptera: Curculionidae). Argania edition, Barcelona, 124 pp.

Derwesh, A. I. 1963. A preliminary list of Coleoptera from Iraq. Directorate General of Agricultural Research and Projects, Technical Bulletin, 13: 1-38.

Derwesh, A. I. 1965. A preliminary list of identified insects and some arachnids of Iraq. Directorate General of Agricultural Research and Projects, Technical Bulletin, 112: $1-123$.

Herling, C., Svendsen, U. G. and Schou, C. 1995. Identification of important allergenic proteins in extracts of the granary weevil (Sitophilus granarius). Allergy Journal, 50(5):441-6.

Ismail, S. I. 2015. Microsculpture exact description of the isolation of some types of beetles weevils family (Curculionidae) in Iraq. Journal of Life Sciences, 9: 526-529.

Kaddou, I. K. 1967. Check-list of some insect fauna of Iraq. Biological Research Center, Publication, 1: 1- 44.

Khalaf, A. N. and Al-Omar, M. A. 1974. A second list of insects of Iraq. Biological Research Center, Publication, 2: 1- 41.

Kuschel, G. 1995. A phylogenetic classification of Curculionoidea to families and subfamilies. Memoirs of the Entomological Society of Washington, 14: 5-33.

Legalov, A. A. 2006. Phylogenetic reconstruction of weevils superfamily Curculionoidea (Coleoptera) using the SYNAP method. Biology Bulletin, 33(2): 127-134. 
Halgurd Rashed Ismael Akrawi and Talal Tahir Mahmoud

Legalov, A. A. 2011. Obzor zhukov-dolgonosikov triby Hyperini (Coleoptera, Curculionidae) Vnutrennej Azii c zamechaniyami po sistematike vidov i opisaniyami novykh taksonov. A review of weevils of thge tribe Hyperini (Coleoptera, Curculionidae) of Inner Asia with remarks on systematic and description of new taxa. Evraziatskij Entomologicheskij Zhurnal / Euroasian Entomological Journal, 10(1): 145-156.

Löbl, I. and Smetana, A. (eds). 2011. Catalogue of Palaearctic Coleoptera. Volume 7. Curculionoidea I. Apollo Books, Stenstrup, 373 pp.

Löbl, I. and Smetana, A. (eds). 2013. Catalogue of Palaearctic Coleoptera. Volume 8. Curculionoidea II. Brill, Leiden and Boston, 700 pp.

Lyal, C. H. C. and Alonso-Zarazaga, M. A. 2006. Addenda and corrigenda to 'A world catalogue of families and genera of Curculionoidea (Insecta: Coleoptera) . 2. Zootaxa, 1202: 21-31.

Marvaldi, A. E., Sequeira, A. S, O'Brien, C. W. and Farrell, B. D. 2002. Molecular and morphological phylogenetics of weevils (Coleoptera, Curculionoidea): do niche shifts accompany diversification. Systematic Biology, 51: 761-785.

May, B. M. 1993. Larvae of Curculionoidea (Insecta: Coleoptera): a systematic overview. Fauna of New Zealand Number 28. Manaaki Whenua Press, Lincoln, Canterbury, $1221 \mathrm{pp}$.

Oberprieler, R. G. 2004. Phylogeny and evolution of the Brachycerinae sensu lato (Coleoptera: Curculionidae). Abstracts CD-ROM, XXII. International Congress of Entomology, Brisbane, 15-21 August 2004. Australian Entomological Society, Brisbane.

Oberprieler, R. G., Marvaldi, A. E. and Anderson, R. S. 2007. Weevils, weevils, weevils everywhere. Zootaxa, 1668: 491-520.

Reitter, E. 1916. Fauna Germanica. Die Käfer des Deutsches Reiches. Nach der analytische Methode bearbeitet. V. Band. K. G. Lutz, Stuttgart, 343 pp.

Salih, I. M. 2007. Taxonomic study of some species of the two subfamilies: Brachyderinae and Cleoninae (Coleoptera, Curculionidae) in some regions of Iraq. Master thesis, College of Basic Education University of Al-Mustansiryiah, 121pp.

Shalaby, F., El- Haidari, H. S. and Derwesh, A. I. 1966. Contribution to the insect Fauna of Iraq (Part I). Directorate General of Agricultural Research and Projects, Technical Bulletin, 143: 1- 11.

Thompson, R. T. 1992. Observations on the morphology and classification of weevils (Coleoptera, Curculionoidea) with a key to major groups. Journal of Natural History, 26: 835-891.

Velázquez de Castro, A. J., Alonso-Zarazaga, M .A. and Outerelo, R. 2007. Systematics of Sitonini (Coleoptera: Curculionidae: Entiminae), with a hypothesis on the evolution of feeding habits. Systematic Entomology, 32: 312-331. 


\section{A survey of weevils (Coleoptera, Curculionoidea)}

Voss, E. 1964. Über neue und bekannte palaearktische Curculionidenarten und -formen, vorwiegend aus der mediterranen Subregion (Coleoptera, Curculionidae) (185. Beitrag zur Kenntnis der Curculioniden). Reichenbachia, 3: 161-171.

Wibmer, G. J. and O'Brein C. W. 1986. Annotated checklist of the weevils (Curculionidae sensu lato) of South America (Coleoptera: Curculionoidea). Memoirs of the American Entomological Institute, 39: 1-563.

Zherichin, V. V. and Egorov, A. B. 1991. Zhuki-dolgonosiki (Coleoptera, Curculionidae) dalnego vostoka SSSR (obzor podsemejstv s opisaniem novykh taksonov). Akademiya Nauk SSSR, Dalnegovostochnoe otdelenie, Vladivostok, 164 pp. 
Halgurd Rashed Ismael Akrawi and Talal Tahir Mahmoud

Bull. Iraq nat. Hist. Mus.

June, (2019) 15 (3): 319-333

مسح لأنواع خنافس السوس رتبة غمدية الاجنحة (Curculionoidea) في بعض مناطق اقليم كوردستان العراق، مع تسجيل انواع جديدة للمجموعة الحشرية العراقية العافية

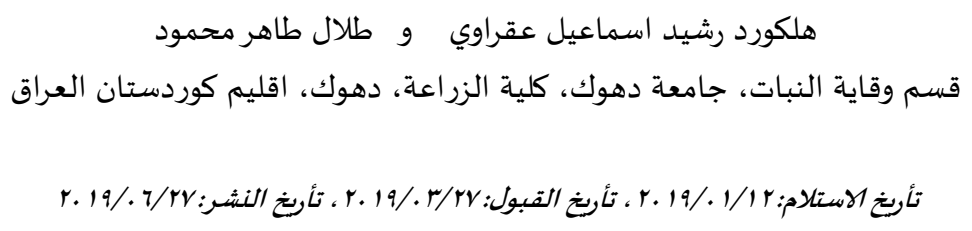

هذا العمل هو أول دراسة شاملة على خنافس السوس في اقليم كوردستان العراق، اعتمادا

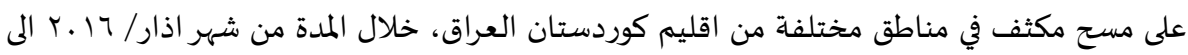

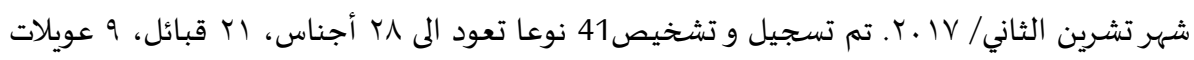

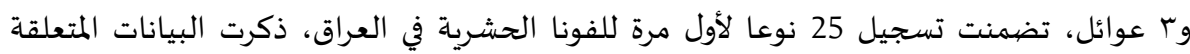
بتوزيع المناطق وطرق الجمع لكل نوع مع النباتات المتواجدة عليها. 